\title{
Análisis empírico de la Hipótesis de Equivalencia Ricardiana para México
}

\author{
Empirical Analysis of the Ricardian Equivalence Hypothesis for Mexico
}

Michel Eduardo Betancourt Gómez* "

Resumen

El ensayo aporta un análisis teórico y empírico sobre el efecto neutral que tiene un déficit público en relación al consumo individual, teoría que se conoce como la Hipótesis de Equivalencia Ricardiana. El análisis teórico resume las diversas controversias que esta teoría ha generado entre los economistas y su confrontación con las teorías clásica y keynesiana. El análisis empírico aporta una evaluación del cumplimiento de la hipótesis para el caso de México y se contrasta frente al modelo de consumo keynesiano. Se concluye que para el caso de México la hipótesis no se cumple en el largo plazo, y en el corto plazo no se cuenta con una línea específica para afirmar o desechar esta concepción, al menos en su versión más débil. Para el caso del contraste keynesiano se concluye que se cumple en ambas especificaciones temporales; por lo tanto, y de manera general, se concluye que los consumidores mexicanos no tienen en cuenta la forma de financiamiento público al momento de consumir.

\section{Palabras clave:}

- Política monetaria

- Mercados financieros

- Predicción y simulación
Abstract

The trial provides theoretical and empirical analysis on neutral effect that has a deficit in relation to individual consumption, theory known as Ricardian Equivalence Proposition. Theoretical analysis summarizes the various controversies that this theory has generated among economists and their confrontation with classical and Keynesian theories. The empirical analysis provides an assessment of compliance of the hypothesis for the case of Mexico and is contrasted against the Keynesian consumption model. We conclude that in the case of Mexico the hypothesis does not hold in the long term, and in the short term there is not a specific line to affirm or reject this concept, at least in its weaker version. To contrast the Keynesian case is concluded that both specifications are met in time; therefore, and in general, it is concluded that Mexican consumers do not take into account the form of public financing at the time of consuming.

\section{Keywords:}

- Monetary Policy

- Forecasting and Simulation

- General Financial Markets

JEL: E52, E17, G1

\section{Introducción}

El debate sobre la función y utilidad del gasto público ha sido amplio durante las últimas décadas, los argumentos van desde tener un objetivo de gasto responsable y una orientación que dé prioridad a un equilibrio de las finanzas públicas, que supone la igualdad entre los ingresos y los egresos presupuestarios; hasta argumentos a favor de tener una política de gasto gubernamental optima para fomentar la demanda, que supone la reducción de impuestos financiada mediante déficit.

Dentro de los argumentos en pro de un equilibrio de las finanzas públicas se encuentra la Hipótesis de Equivalencia Ricardiana (HER) la cual es el objeto de estudio en este ensayo, esta es una particularidad teórica sobre los 
efectos de un déficit público en relación al consumo individual. Esta hipótesis supone que para el consumidor el déficit público equivale a impuestos futuros. Por lo tanto, es un argumento comúnmente utilizado para afirmar lo necesario que es un equilibrio presupuestal en los gastos del gobierno.

La razón de este ensayo es determinar empíricamente si esta hipótesis se cumple para el caso de México, y justificar así la orientación ideológica del gobierno por el equilibrio fiscal. Por tal motivo se realiza un contraste entre la hipótesis ricardiana y la teoría keynesiana, ya que esta ultima supone un efecto positivo en el consumo ante un aumento del gasto público, por lo que su comparación puede determinar si dicho gasto fomenta el consumo, como argumenta el modelo keynesiano; o su efecto es neutral, y la orientación de equilibrio fiscal es justificada. Este análisis empírico se hace bajo el enfoque de la teoría de la cointegración de Granger (1987), lo que permite revisar el comportamiento de las variables tanto en el largo plazo como en el corto plazo.

En el primer apartado de este ensayo se presenta una discusión teórica acerca de la Equivalencia Ricardiana, dentro de esta se dan argumentos que contrastan la hipótesis con las teorías clásica y keynesiana. Debido a que los autores mencionados en este apartado consideran ciertas características particulares de una economía nacional para que esta teoría no pueda cumplirse, el segundo apartado del ensayo es una justificación para el caso de México de los aspectos de la economía que pudieran distorsionar el análisis empírico.

El tercer apartado es el análisis empírico de la Equivalencia Ricardiana para el caso de México: se hace una revisión de literatura de las distintas evaluaciones empíricas de la hipótesis y se plantea una función de consumo estructural para evaluar el cumplimiento de la teoría. Después se realiza un contraste entre las dos concepciones teóricas del gasto público en relación al consumo: la keynesiana y la de Equivalencia Ricardiana (en su versión fuerte y débil), esto, para determinar cuál es significativa para el caso de México.

\section{Disertaciones teóricas sobre la Hipótesis de Equivalencia Ricardiana}

Existen diversas concepciones teóricas acerca del papel que representa la deuda pública para alguna economía nacional. La teoría tradicional de la deuda pública supone que cuando el Gobierno baja los impuestos e incurre en un déficit presupuestario, los consumidores responden al aumento del ingreso neto (ingreso después de impuestos) gastando más. Por lo tanto, un 
déficit público eleva la demanda agregada y aumenta la producción a corto plazo, pero reduce el capital y el crecimiento económico a largo plazo.

En el caso de la teoría keynesiana, un aumento del gasto público financiado mediante deuda genera un efecto multiplicador en el ingreso de los consumidores, la razón de esto se debe a la concepción de la función de consumo ${ }^{1}$. Por lo tanto, un mayor ingreso genera un mayor consumo, lo que aumenta la demanda agregada, la producción y el crecimiento económico.

La tercera visión acerca de la deuda pública y que es de interés particular para este ensayo es la Hipótesis de Equivalencia Ricardiana ${ }^{2}$, ya que pone en controversia los efectos de la teoría tradicional y la keynesiana. Según la Hipótesis de Equivalencia, un déficit público no produce ninguno de los efectos mencionados en las teorías anteriores, porque supone que los consumidores comprenden que solo representa el retraso de una carga tributaria futura. Es decir, los individuos son previsores y, en consecuencia, basan su gasto no solo en su ingreso actual sino en el ingreso que esperan percibir en el futuro.

La HER aplica la lógica del consumidor racional ante variaciones temporales de la política fiscal. Ante la posibilidad de gasto deficitario del gobierno, el consumidor, de manera precavida, decide ahorrar al evaluar la posibilidad que en el futuro el gobierno tenga que subir impuestos para compensar el déficit en el presente, por lo tanto, un aumento del déficit no altera el consumo de las personas. En otras palabras, el consumidor previsor comprende que el endeudamiento público actual significa unos impuestos más altos en el futuro. Una reducción de los impuestos financiada mediante deuda pública no reduce la carga tributaria; simplemente, la retarda. Por lo cual no debería animar al consumidor a gastar más, sino a ahorrar para pagar una subida inevitable de impuestos en el futuro.

Lo anterior supone que una reducción de los impuestos financiada mediante deuda no afecta el consumo. Los hogares ahorran el ingreso disponible adicional para pagar la futura deuda tributaria consecuencia de la reducción de impuestos. Este incremento en el ahorro privado, compensa exactamente la disminución del ahorro público. Por tanto, la reducción de impuestos no tiene ninguno de los efectos que los argumentos teóricos tradicional o keynesiano sustentan.

\footnotetext{
${ }^{1}$ La tradicional función de consumo keynesiana postula que el consumo individual depende del ingreso disponible. Keynes (1936) sostenía que el volumen del consumo agregado, depende del ingreso agregado.

${ }^{2} \mathrm{El}$ nombre se deriva del economista David Ricardo, quien fue el primero en proponer esta idea, aunque finalmente la desechó. Para revisar la discusión completa ver Ricardo (1820): "Essay on the Funding System".
} 
Cabe señalar que la lógica de la Equivalencia Ricardiana no significa que todos los cambios en la política fiscal son irrelevantes. Los cambios en la política fiscal si logran que el consumidor influya en las compras actuales o futuras del gobierno. Por ejemplo, "supongamos que el gobierno reduce los impuestos de hoy, ya que planea reducir el gasto gubernamental en el futuro. Si el consumidor entiende que este recorte de impuestos no requiere un aumento en los impuestos futuros, se siente más rico y eleva su consumo. Pero hay que notar que es la reducción en el gasto público, en lugar de la reducción en impuestos, lo que estimula el consumo: el anuncio de una futura reducción en las compras de gobierno elevaría el consumo de hoy en día, aunque los impuestos corrientes se mantengan sin cambios, ya que implicaría la reducción de impuestos en algún momento en el futuro.” (Mankiw, 1995).

En esencia, la Hipótesis de Equivalencia asume que cuando los individuos eligen su nivel de consumo, racionalmente consideran impuestos implícitos futuros en la deuda pública, por lo que no consideran en la deuda pública o en la reducción impositiva una fuente de riqueza personal. Análisis teóricos de diversos economistas como Bernheim (1987), Mankiw (1995) y Romer (2006) muestran algunas deficiencias sobre la HER con respecto a la teoría tradicional de la deuda pública o la concepción del gasto público en la teoría keynesiana.

\section{Análisis teóricos de Mankiw, Romer y Barro}

En su análisis, Mankiw (1995) argumenta que los defensores de la visión tradicional de la deuda del gobierno creen que la perspectiva de futuros impuestos no tiene una influencia tan grande en el consumo actual como la Hipótesis de Equivalencia asume. Éstos son algunos de sus argumentos:

I) Miopía: Los defensores de la HER suponen que las personas son racionales al tomar decisiones como qué cantidad de sus ingresos destinan a consumir y cuánto a ahorrar. Cuando el gobierno pide prestado para pagar el gasto corriente, la racionalidad de los consumidores hace que miren al futuro para calcular los impuestos necesarios para apoyar esta deuda. Así, la Equivalencia Ricardiana presume que las personas tienen un conocimiento sustancial y de previsión a la hora de tomar sus decisiones de consumo y ahorro.

Pero un argumento posible que establece la visión tradicional de los recortes de impuestos es que la gente es miope, tal vez porque no comprenden plenamente las consecuencias de los déficit presupuestarios del gobierno. 
Es posible que algunas personas sigan reglas simples y no totalmente racionales al elegir cuánto ahorrar o cuánto consumir. "Supongamos, que una persona actúa creyendo que los impuestos futuros serán los mismos que los impuestos actuales. Esta persona va a dejar de tener en cuenta los futuros cambios en los impuestos requeridos por las políticas gubernamentales actuales. Un recorte de impuestos financiado con deuda dará lugar a esta persona a creer que su ingreso de por vida ha aumentado, aunque no lo ha hecho. Por tanto, la reducción de impuestos dará lugar a un mayor consumo y menor ahorro nacional.”(Mankiw, 1995). La conclusión del supuesto, radica en considerar que si el consumidor es miope, la visión tradicional y keynesiana de la deuda pública puede cumplirse.

II) Mercado de Capitales Imperfectos/Restricciones Crediticias ${ }^{3}$ : La HER parte del supuesto de que los consumidores basan su gasto no solo en su ingreso actual sino también en su ingreso permanente, que comprende tanto el ingreso actual como el ingreso esperado. De acuerdo con la Hipótesis de Equivalencia, una reducción de los impuestos financiada mediante deuda eleva el ingreso personal actual, pero no altera el ingreso obtenido a lo largo de la vida y el consumo realizado durante todo ese tiempo. Los defensores de la teoría tradicional de la deuda pública sostienen que el ingreso actual es más importante que el ingreso obtenido a lo largo de la vida para los consumidores que se enfrentan a restricciones crediticias.

Las personas a las que les gusta consumir más de lo que les permite su ingreso actual -debido tal vez a que esperan percibir un ingreso más alto en el futuro- tienen que pedir un crédito. Si no pueden pedirlo para financiar su consumo actual o solo pueden solicitar una cantidad limitada, su ingreso actual determina su gasto de consumo, independientemente de cuál sea el ingreso que se obtengan a los largo de toda su vida. En este caso, una reducción de los impuestos financiada mediante deuda eleva el ingreso actual y, por lo tanto, el consumo, aun cuando el ingreso futuro sea más bajo.

Cuando el gobierno baja los impuestos actuales y eleva los futuros, concede un préstamo a los contribuyentes. Para una persona que quiera conseguir un préstamo y no pueda, la reducción de los impuestos aumenta sus oportunidades y estimula el consumo. Es por este razonamiento que los defensores de las teorías tradicional y keynesiana asumen que un aumento del déficit aumenta el consumo: porque para los individuos el ingreso actual es más importante que el ingreso obtenido a lo largo de su vida, es decir de su ingreso permanente.

\footnotetext{
${ }^{3}$ Las restricciones crediticias se refieren a una limitación de la cantidad que puede pedir prestada una persona a los bancos o a otras instituciones financieras. Para profundizar con respecto a la HER revisar: Mankiw (1995), Bernheim (1987) y Romer (2006).
} 
Este debate acerca del ingreso permanente en relación a la HER lo analiza también Romer (2006). Él contrasta la Equivalencia Ricardiana desde la óptica de la Hipótesis del Ingreso Permanente de Friedman ${ }^{4}$. Como asume Mankiw (1995), la HER parte del supuesto de que los individuos basan su consumo en su ingreso permanente aparte de su ingreso actual, por lo tanto Romer se da cuenta que el análisis de la Equivalencia Ricardiana pasa por el análisis de la Hipótesis de Friedman. Es decir, la HER depende de la Hipótesis del Ingreso Permanente, y si esta ultima presenta fallos, podría desestimar en su totalidad el razonamiento ricardiano.

Romer (2006) argumenta que: "en el modelo de la renta (ingreso) permanente, lo único que afecta al consumo de una economía domestica es su restricción presupuestaria a lo largo del ciclo vital; la evolución temporal de su renta neta no tiene importancia. Una emisión de bonos en el presente que se pague mediante impuestos futuros únicamente afecta a la evolución de la renta disponible pero no la restricción presupuestaria a lo largo del ciclo de vida. De modo que si la hipótesis de la renta permanente es una buena descripción del comportamiento del consumo, es probable que la Equivalencia Ricardiana sea también una buena aproximación. Pero si existen desviaciones significativas respecto de la hipótesis de la renta permanente, es probable que tampoco se cumpla la Equivalencia Ricardiana”.

Entonces para Romer la viabilidad del cumplimiento de la Equivalencia Ricardiana recae en la concepción de la Hipótesis del Ingreso Permanente, ya que, asume que el consumo de los individuos depende también de su ingreso a lo largo de su ciclo de vida. $\mathrm{El}$ argumenta dos posibilidades donde no se cumple la Hipótesis de Ingreso Permanente: la primera es la misma descrita por Mankiw (1995), y son las restricciones de liquidez: "cuando el gobierno entrega a una familia un bono que más tarde pagará con mayores impuestos, lo que de hecho está haciendo es tomar prestado en nombre de la familia. Si la economía domestica todavía tiene la opción de pedir prestado al mismo tipo de interés del gobierno, la política no tiene efecto sobre sus oportunidades y, por tanto, tampoco sobre su comportamiento. Pero supóngase que la economía domestica se enfrenta a un tipo de interés superior al del gobierno y que si la economía domestica pudiera pedir prestado a este ultimo tipo de interés para aumentar su consumo lo haría. En estas condiciones, la respuesta del hogar al préstamo que ha tomado el gobierno

${ }^{4}$ Friedman (1957) afirmó que la concepción de la función del consumo agregado de Keynes estaba equivocada y concluyó que el consumo solo depende del ingreso permanente, que es la parte del ingreso que los individuos esperan que persista en el futuro. Este argumento se conoce como la Hipótesis del Ingreso Permanente. 
en su nombre seria elevar su nivel de consumo." Romer (2006). Al existir dichas restricciones de liquidez, los consumidores van a optar por aumentar su consumo a costa del ingreso adicional que genera la reducción impositiva o el aumento del gasto vía déficit publico. Romer concluye que si las restricciones de liquidez son causa de fallos importantes a la Hipótesis de Ingreso Permanente, es probable que el comportamiento de la economía se desvié considerablemente de lo que prevé la Equivalencia Ricardiana.

La segunda causa del incumplimiento de la hipótesis del ingreso permanente y que puede desestimar la HER es la concepción del ahorro precautorio y una elevada tasa de descuento. Con la emisión de deuda, las futuras obligaciones impositivas de los individuos serán solo ligeramente superiores si su ingreso llegara a ser bajo. Es decir, "la combinación de una reducción de impuestos hoy y un aumento de impuestos en el futuro eleva el valor presente del ingreso disponible a lo largo del ciclo de vida de la familia si su ingreso futuro es bajo y lo reduce en caso contrario." (Romer, 2006).

En consecuencia, los individuos tienen pocos incentivos para aumentar su ahorro. En vez de eso, puede permitirse guiarse por su elevada tasa de descuento y aumentar su consumo sabiendo que sus futuras obligaciones impositivas solo serán elevadas si lo es también el ingreso. Por lo tanto, al contrastar el análisis de Romer y de Mankiw acerca de las restricciones crediticias o de liquidez con la HER, puede cumplirse la teoría tradicional o keynesiana del gasto público: al existir estas restricciones al ingreso de los individuos, y estos al querer consumir más y carecer de crédito para aumentarlo, o al pensar que los impuestos futuros serán mayores solo si su ingreso futuro lo es, el aumento de su ingreso proveniente de la reducción de impuestos (o el aumento del déficit) actúa como incentivo para consumir, desalentando el ahorro; razón por la cual la HER no puede cumplirse.

III) Generaciones Futuras: este tercer apartado está a favor de la visión tradicional de la deuda pública y argumenta que los consumidores esperan que los impuestos futuros que implica esta no recaigan en ellos sino en las generaciones futuras. Es decir, si el gobierno baja hoy los impuestos, emite bonos a cierto número de años para financiar el déficit presupuestario y, en consecuencia, sube los impuestos dentro del mismo número de años para devolver el préstamo. "En este caso, la deuda pública representa una transferencia de riqueza de la siguiente generación de contribuyentes (que se enfrentan a una subida de los impuestos) a la generación actual durante toda su vida, por lo que eleva su consumo." (Mankiw, 1995). 
Resumiendo, este argumento de los defensores de la teoría tradicional, afirma que una reducción de los impuestos financiada mediante deuda estimula el consumo porque brinda a la generación actual la oportunidad de consumir a expensas de la generación siguiente. Por lo tanto, este argumento, también contribuye a sustentar el modelo keynesiano porque indica que el consumo se incrementa ante una subida del gasto público.

$\mathrm{Al}$ respecto de este último razonamiento que desaprueba en parte la $\mathrm{Hi}$ pótesis de Equivalencia en favor de la visión clásica del efecto de la deuda pública, el economista Robert Barro (1974) dio una respuesta en apoyo de la HER. Barro sostiene que como las generaciones futuras son los hijos y los nietos de la generación actual, no debemos considerarlas como agentes económicos independientes. Sostiene que lo que hay que suponer es que las generaciones actuales se preocupan por las futuras.

Este altruismo lo demuestran las donaciones que hacen muchas personas a sus hijos, a menudo en forma de herencias en el momento de su defunción. La existencia de donaciones parece indicar que mucha gente no desea aprovechar la oportunidad de consumir a costa de sus hijos.

De acuerdo con el análisis de Barro (1974), la unidad relevante para tomar decisiones no es la persona, que solo vive un numero finito de años, sino la familia, que pervive. En otras palabras una persona decide cuánto va a consumir basándose no solo en su propio ingreso sino también en el de los futuros miembros de su familia. Una reducción de los impuestos financiada mediante deuda puede elevar el ingreso que percibe una persona a lo largo de su vida, pero no aumenta los recursos totales de su familia. Entonces, en lugar de consumir el ingreso adicional generado por la reducción de los impuestos, el individuo lo ahorra y lo deja en herencia a sus hijos, que tendrán que hacer frente a la futura subida inevitable de impuestos.

Con esto, queda establecido que el debate teórico de la deuda pública, se resume en parte, en un debate sobre la conducta de los consumidores. La HER supone que estos tienen un horizonte temporal de largo plazo. El análisis de la familia que hace Barro supone que el horizonte temporal del consumidor, como el del Estado es, de hecho, infinito. Sin embargo, es posible que los consumidores no tengan en cuenta las obligaciones tributarias de las futuras generaciones. Quizá esperan que sus hijos sean más ricos que ellos y, por lo tanto, se alegren de la oportunidad de consumir a costa de sus hijos. El hecho de que muchas personas dejen una herencia nula o mínima a sus hijos es coherente con esta hipótesis. En el caso de estas familias que no dejan ninguna herencia, una reducción de los impuestos financiada 
mediante deuda altera el consumo redistribuyendo entre las generaciones (Barro, 1974).

\section{Análisis teórico de Bernheim}

$\mathrm{El}$ argumento de Barro sobre las generaciones infinitas ha generado controversia entre los economistas, en el análisis de Bernheim (1987) se contempla el debate de varios autores en relación con la HER. Sostiene que él (Bernheim, 1986) y varios autores más (Poterba y Summers (1986), Hubbard y Judd (1986)) divergen sobre la contundencia del argumento de las generaciones futuras y las consideraciones de los consumidores sobre estas, ellos afirman que existe la posibilidad que una fracción sustancial de la carga de los impuestos no se desplace a las generaciones futuras, como el argumento de Barro sostiene, y además mencionan que cuando el déficit actúa de manera atípica, los consumidores racionales bien podrían esperar eludir su responsabilidad para el pago de la mayor parte de la deuda actual. Los autores terminan concluyendo que factores tales como las restricciones de liquidez y la miopía tienen una influencia mucho mayor en el debate de la HER.

En el mismo análisis, Bernheim (1986) considera distintos y estrictos supuestos que dependen de la Hipótesis de Equivalencia para que pueda cumplirse; su contrastación es similar al análisis de Mankiw (1995) pero la diferencia entre ambos es que Bernheim considera, además, los siguientes aspectos:

I) La redistribución impositiva: Se asume que un diferimiento social de impuestos, es decir, una política fiscal progresiva, a través de un déficit público puede alterar el patrón de incidencia de estos en el consumo de los individuos. Es decir, diferentes tipos de personas pueden tener una mayor participación en la carga impositiva en diferentes momentos, esto puede alterar significativamente el consumo de los individuos y por lo tanto la concepción principal de la HER.

II) Distorsión impositiva: La dinámica fiscal puede ser muy importante si los impuestos llegan a distorsionar el comportamiento, y si la tasa marginal impositiva temporal depende de diferentes decisiones de los impuestos corrientes. Esta observación es especialmente relevante a la discusión de la HER cuando se utiliza el déficit para posponer los impuestos a los ingresos de capital por ejemplo, ya que una reducción de las tasas de dichos impuestos puede hacer que los individuos tiendan a ahorrar más y consumir menos. Por eso la selección de impuestos por parte del Gobierno puede alterar el comportamiento de consumo y ahorro de los individuos. 
III) Aplazamiento permanente: Es un argumento que en la actualidad tiene mucho auge, supone que puede existir un aplazamiento permanente de impuestos debido a que la tasa de crecimiento de una economía supera a la tasa de interés, por lo tanto el déficit no afectaría al gobierno, aunque si pudiera afectar la racionalidad de los consumidores, ya que, en realidad nunca pagarían su suposición del aumento de impuestos, esto generaría un aumento considerable de su ahorro.

Feldstein (1976) criticó el análisis de la política fiscal de Barro con el argumento anterior: cuando la tasa de crecimiento de la economía supera a la tasa de interés, el gobierno puede revertir el déficit indefinidamente. Como resultado, ninguna generación necesita pagar la porción de los impuestos aplazados. Barro (1976) respondió que los vínculos intergeneracionales en realidad podrían evitar que exista una ineficiente sobreacumulación de capital.

Aunque en realidad no se puede concluir la veracidad de ambos enfoques, es importante resaltarlos: el primero es que el altruismo intergeneracional no descarta resultados temporales ineficaces, y el segundo el referente al exceso de capital en una economía.

Como se puede notar, el debate de la Hipótesis de Equivalencia Ricardiana versa sobre tres diferentes aspectos: en primer lugar, las consideraciones de la conducta de los individuos, racional y previsora; en segundo lugar, sobre como las generaciones están vinculadas entre sí, es decir, las familias se comportan como dinastías para perpetuarse infinitamente; y en tercer lugar la dinámica impositiva, que quiere decir cómo afecta la determinación de las tasas y los tipos de impuestos al ingreso y al consumo de las economías domesticas. Estos tres aspectos coinciden en un solo asunto: el consumo de los individuos; por lo tanto, todo análisis de la HER debe examinar el comportamiento del consumo para comprobar o no la veracidad de esta hipótesis.

\section{Consideraciones de la HER para el caso de México}

El caso de la economía mexicana tiene ciertas particularidades que pueden sugerir la distorsión de la concepción empírica de la HER. Hay que señalar las características que posee y los contrastes que puede tener para que su comparación sea significativa y valida. Los aspectos más importantes a considerar son: en primer lugar, la dinámica impositiva y el panorama fiscal mexicano; en segundo lugar, la estructura de su sistema financiero, ambos aspectos esenciales para el cumplimiento de la HER. 
El primer aspecto a considerar es el Sistema Fiscal Mexicano, que actualmente está constituido por cuatro impuestos que generan cerca del 63\% de la recaudación impositiva: el impuesto sobre la renta (ISR), empresarial y personal; el impuesto al valor agregado (IVA); el impuesto empresarial a tasa única (IETU); y el impuesto especial sobre producción y servicios (IEPS) que grava la enajenación o importación de diversos bienes y servicios. El nivel de estos impuestos se ha mantenido relativamente constante durante las últimas décadas, por ejemplo, el ISR presentó una reducción pasando de $35 \%$ en 1993 a 30\% en 2012; y para el caso del IVA paso de 10\% en 1993 a 16\% para 2012. Debido a que el nivel de estos impuestos se ha mantenido constante, la recaudación no ha variado significativamente.

Pero a pesar de los avances obtenidos en la estructura del Sistema Fiscal y en la administración tributaria durante las últimas dos décadas, la recaudación fiscal en México presenta diversos problemas: existe una baja recaudación impositiva, en 2012 la recaudación fue de 15\% en proporción al Producto Interno Bruto (PIB), porcentaje muy por debajo del promedio de de los países miembros de la Organización para la Cooperación y el Desarrollo Económicos (OCDE) que es del 25\%; también, algunos impuestos carecen de progresividad o esta es muy tenue, lo que afecta a la redistribución: cerca del 70\% de la contribución del ISR en las personas físicas fue generado por los últimos dos estratos de la población con mayores ingresos.

La principal razón de conocer el panorama impositivo en México es determinar que si con su estructura la HER pudiera no ser compatible por los problemas mencionados en el análisis teórico. Si bien el sistema fiscal presenta carencias y fallas, no afecta la concepción de la HER ya que se usa el mismo razonamiento: los consumidores mexicanos, al no variar los impuestos pero al ser mayor los egresos públicos sustentados en este caso por el déficit público en una parte, pero también por los ingresos petroleros, pensaran en reducir su consumo actual y ahorrar para cuando supongan que el petróleo se acabará y los egresos presupuestarios se tengan que compensar con una subida de impuestos, por lo tanto, no afectaría la esencia de la HER el hecho de que los impuestos en México no varien con el tiempo. ${ }^{5}$

Otro aspecto a destacar es la cuestión de la redistribución impositiva que para el caso mexicano no es tan acentuada, ya que al carecer de cierta progresividad en los impuestos, no llega a generar un problema para des-

${ }^{5}$ Es importante mencionar que existe un área de debate para esta afirmación, pero para efectos de este ensayo se asume que los ingresos petroleros tienen la misma función que el déficit público: ambos reducen la carga tributaria de los individuos y en consecuencia su ingreso no se ve reducido; un análisis más completo sobre tal concepción va mas allá del alcance de este ensayo. 
estimar la concepción de la HER. Lo mismo pasa con la distorsión impositiva, ya que los impuestos tributarios, al depender mayoritariamente de solo cuatro impuestos se nota que no hay una diversificación por el poco interés gubernamental por ampliarlos y aumentar la recaudación; esto debido a la existencia de los ingresos provenientes del petróleo, que representan un gran porcentaje de los ingresos públicos totales.

El segundo aspecto que puede distorsionar el consumo mexicano, y por lo tanto la concepción de la HER, es el caso de la estructura del Sistema Financiero Mexicano, ya que una parte importante de la HER mantiene el supuesto de un ahorro continúo en caso de que el déficit sea elevado para compensar la futura subida de impuestos.

Actualmente el Sistema Financiero Mexicano es limitado, el ahorro en bancos representa menos de $10 \%$ del PIB, y solo entre 15 y $25 \%$ de la población urbana, y 6\% de la población rural tiene acceso a cuentas en instituciones financieras. Estas limitaciones generan ciertas asimetrías para la adquisición de servicios financieros, tales como el ahorro y el crédito.

Estas asimetrías pueden distorsionar el consumo de los individuos, ya que debido al corto alcance que tienen las instituciones financieras para proveer servicios que lleguen a todos los estratos de la población, en México se tienden a ocupar formas informales y hasta rudimentarias de ahorro, por ejemplo. Pero algo que hay que resaltar es que a pesar de las restricciones de liquidez que posee la mayor parte de los mexicanos que viven en zonas dispersas, ahorran, aunque en pequeñas cantidades y su ahorro solo es para el corto plazo. Las formas actuales de servicios financieros para este tipo de población han hecho notar que hay una ligera tendencia de ahorro en el mexicano con restricciones de liquidez, lo que evidencia su interés por ahorrar, esto por lo tanto no genera un posible problema para el análisis de la HER en el caso mexicano. ${ }^{6}$

Debido a que los servicios financieros no llegan a la mayor parte de la población, puede ocasionar una distorsión no solo en el ahorro de los individuos, sino también un problema en el acceso al crédito que requieren para aumentar su consumo. Como se argumenta en la discusión teórica de este ensayo, las restricciones crediticias pueden afectar la concepción de la HER ya que, al existir pueden generar una limitación de los individuos al crédito lo que puede estar siendo compensado por las transferencias de los egresos

${ }^{6}$ El estudio del ahorro en México es limitado y poco concluyente, en general se ha investigado empíricamente la tendencia a ahorrar del mexicano con restricciones de liquidez y se ha demostrado que este ahorra para reducir dichas restricciones y no caer en diversos estratos de pobreza. Para un análisis al respecto revisar Villagomez (2008): "El ahorro en México desde 1960: estructura, evolución y determinación". 
presupuestarios. Pero como el crédito al consumo en el caso de México ha venido en aumento durante la última década, en parte porque es el crédito más rentable, no puede ocasionar una gran distorsión para poder contrastar la HER de manera confiable.

\section{Análisis empírico de la HER para México}

El análisis empírico aporta la evidencia del comportamiento real de los datos y puede contribuir a verificar la certeza que tiene la HER. El tema principal de estudio en este ensayo es analizar empíricamente la veracidad de la Hipótesis de Equivalencia para el caso mexicano. Es decir, evaluar el comportamiento del consumo en México en relación al déficit, por lo que en esta revisión de literatura solo se consideran aquellos análisis empíricos que tengan una orientación hacia la estimación de una función estructural del consumo y su contrastación con la HER.

Kochin (1974) realizó uno de los primeros trabajos empíricos sobre la HER. Lo hace definiendo una función de consumo cuya formulación teórica parte de la hipótesis del ingreso permanente. De manera específica, Kochin introduce el déficit público como variable explicativa en una función de consumo dependiente del ingreso disponible. Con datos de Estados Unidos para el periodo de 1952 a 1971, estima un coeficiente de la variable déficit, obteniendo un coeficiente significativo y negativo, aunque inferior respecto al coeficiente del ingreso. Kochin concluye que los individuos si han tenido en cuenta el gasto gubernamental al consumir y al ahorrar. Este análisis respalda, aunque en una versión muy débil, la HER.

Posteriormente Yawitz y Meyer (1976) realizan un análisis que pretende ampliar las conclusiones de Kochin. Para ello especifican una función de consumo con una justificación teórica proveniente de la Hipótesis del Ciclo Vital de Ando-Modigliani ${ }^{7}$. Es decir, la función de consumo que estiman depende del ingreso disponible, de la cartera de deuda del sector privado a precios de mercado, y de la riqueza (patrimonio) privada neta antes de la deuda. Los resultados obtenidos para Estados Unidos en el período 1953-1967 no apoyan la existencia de un descuento o anticipo impositivo, y concluyen en su análisis que la deuda pública es percibida como riqueza neta.

\footnotetext{
${ }^{7}$ Durante la década de 1950 Franco Modigliani y Albert Ando, estudiaron la relación de consumo de los individuos. Propusieron la Hipótesis del Ciclo Vital, la cual supone que las personas administran su ingreso para mantener su nivel de consumo constante a lo largo de su vida. Por ello argumentaron que los individuos ahorran para utilizar esos recursos en periodos de vida difíciles. Concluyen que los individuos ahorran cuando su ingreso es alto, y desahorran cuando dejan de trabajar.
} 
Buiter y Tobin (1980) criticaron el trabajo de Kochin y propusieron su propia función de consumo estructural, misma que contenía como variables independientes el ingreso nacional, los impuestos, el déficit y el consumo desfasado. Al aplicar la estimación para Estados Unidos, en el periodo 19491979, concluyen que la HER no se justifica porque no es significativo el parámetro del déficit público, aunque afirman que es necesaria una investigación empírica mayor para obtener contundencia en los resultados que obtuvieron.

De manera indirecta, Feldstein (1982) analiza la neutralidad fiscal que argumenta la HER. Para él, la eficacia de la política fiscal depende de la forma en que el consumidor vincula un cambio fiscal a sus futuras obligaciones impositivas y cómo esas obligaciones impositivas afectan su comportamiento temporal. En específico Feldstein estima una función de consumo donde relaciona el consumo individual con el ingreso disponible, la riqueza y variables fiscales como el gasto público, ingresos tributarios, entre otras (utiliza las variables en términos reales). Los resultados de sus estimaciones para Estados Unidos en el período 1930-1976, sugieren que la HER no tiene soporte porque los cambios en las tasas impositivas o en gasto público pueden tener efectos sustanciales sobre la demanda agregada.

Por otro lado, Kormendi (1983) estima una función de consumo similar al del modelo de Kochin y Buiter-Tobin. La función de consumo estructural que estima depende del ingreso nacional neto, el regazo de ese mismo ingreso, el gasto público total (consumo de gobierno mas el gasto de inversión), los ingresos fiscales totales, la deuda pública, las transferencias y la riqueza privada sin incluir la deuda, los beneficios empresariales no distribuidos y el pago de los interés del gobierno. Los resultados obtenidos del análisis apoyan el planteamiento de la HER: los parámetros estimados muestran que un incremento en los impuestos no afecta el consumo privado mientras que un aumento en el gasto público lo reduce; asimismo obtiene un coeficiente asociado a la deuda pública no significativamente positivo. ${ }^{8}$

Para el caso de Europa, Raymond y González-Paramo (1987) estiman una función de consumo estructural que depende del consumo desfasado, el ingreso disponible, impuestos, transferencias, gasto público y déficit público (utilizan estas variables en términos per cápita). Esta especificación, está inspirada en la función de consumo propuesta por Buiter-Tobin, y sus

\footnotetext{
${ }^{8}$ Sin embargo, Feldstein y Elmendorf (1987) replicaron el trabajo de Kormendi utilizando su misma especificación econométrica, aunque excluyendo años específicos que, a razón de los autores, alteraron el comportamiento del consumo y el ahorro en Estados Unidos. Esto debido a la Segunda Guerra Mundial, en la cual la escasez, el racionamiento y la retorica patriótica para el autoconsumo causaron una cantidad anormalmente alta del ahorro, al mismo tiempo el déficit del gobierno aumento excepcionalmente por los gastos de defensa. Al prescindir de esos años, Feldstein y Elmendorf llegaron a la conclusión opuesta a la de Kormendi.
} 
autores argumentan que tiene la ventaja de anidar como casos especiales la función keynesiana de consumo y la HER.

Raymond y González-Paramo contrastan ambas posturas teóricas para el caso especifico de la economía española en el periodo de 1955 a 1986 y concluyen que la Hipótesis de Equivalencia, en su versión más estricta de neutralidad fiscal, contradice a los datos, además, su capacidad explicativa es inferior al que se deriva del enfoque keynesiano; por lo tanto, concluyen que la concepción keynesiana del consumo en España es más significativa que la HER.

Posteriormente, Fuster (1993) retoma parte de lo hecho por RaymondGonzález-Paramo y Buiter-Tobin, la diferencia radica en el método de estimación y ligeras adiciones a la función de consumo estructural que sustenta con argumentos teóricos. Fuster, en vez de realizar estimaciones individuales, realiza una general que contrasta la HER, frente el modelo keynesiano, para cinco países pertenecientes a la Comunidad Europea: España, Italia, Alemania, Francia y Reino Unido, en el periodo 1964-1988.

Los resultados que Fuster (1993) encuentra son dispares: para ninguno de los países de estudio comprueba la contundencia estricta de la HER, pero indica el caso de Reino Unido, que concluye se aproxima a la concepción keynesiana. No obstante, aunque no demuestra el cumplimiento de la Hipótesis de Equivalencia de manera estricta para los demás países, afirma que en general los consumidores tienen en cuenta las decisiones de financiamiento público a la hora de tomar decisiones.

Como se puede notar, la mayoría de los análisis empíricos realizados provienen de la especificación de la función de consumo estructural propuesta por Buiter y Tobin (1980). Siguiendo dicho análisis, y las ampliaciones hechas por Raymond-González-Paramo (1987) y Fuster (1993), en este ensayo se propone un análisis empírico de la HER para el caso de México partiendo de la función de consumo estructural propuesta y ampliada por estos autores. Las diferencias principales a estos modelos, es que solo se evalúa el comportamiento del consumo mexicano, por lo que este análisis es solo para un país, al contrario de los trabajos de Fuster, además, el método de estimación propuesto diverge del utilizado por Raymond-González-Paramo y Buiter-Tobin.

\section{Modelo de estimación}

\section{de la función de consumo estructural para México}

El modelo de estimación general que se propone en este ensayo es la ampliación que hace Fuster. "Se trata de una función de consumo sencilla a la que se han añadido aspectos dinámicos y variables precio. En dicha función se 
han considerado como variables explicativas el ingreso disponible de las familias (disociada en sus tres componentes) y el déficit público (para que sea posible comprobar si el consumo se ve afectado por las decisiones de política fiscal). La variable dependiente se expresa en incrementos con el objeto de que tanto el coeficiente de determinación como la capacidad de ajuste del modelo tengan una interpretación más clara.” Fuster (1993).

Para el análisis de la estimación se hacen diversos cambios al modelo ampliado de Fuster para adaptarlo al caso de México: en primer lugar, no incluye la variable transferencias, que es uno de los componentes del ingreso privado, la razón de esto se debe a que para el caso mexicano no existe información sobre este apartado, pero se asume que este componente ya viene expresado en el ingreso, por lo tanto, quitar esta variable no afecta la composición general del modelo de estimación. En segundo lugar, en vez de usar la variación de precios, se utiliza directamente la inflación, esto, por la periodicidad que para el caso mexicano se hace sobre el Índice de Precios al Consumidor (INPC), al tomar la Inflación anual, mejora la eficiencia de la estimación.

Por lo anterior, el modelo general de la función de consumo para México que sirve como punto de partida para el contraste de las especificaciones ricardianas (versión fuerte y débil) y keynesianas es el siguiente:

$$
\Delta C P_{t}=\alpha_{0}+\alpha_{\mathrm{i}} C P_{t-4}+\alpha_{2} Y_{t}+\alpha_{3} I P_{t}+\alpha_{4} D P_{t}+\alpha_{5} \pi_{t}+U_{t}
$$

Donde CP representa el consumo privado, $\mathrm{Y}$, los ingresos de las economías domesticas antes de impuestos, IP, los ingresos públicos, DP, el déficit del gobierno y $\pi$ la inflación.

Fuster (1993) y Brett Inder (1993) defienden la inclusión de la variable endógena desfasada como regresor de la ecuación de largo plazo, por que argumentan que se espera con esto mejorar la eficiencia de la estimación. Señalan que, aun en el caso de que las variables estén cointegradas, la eliminación de la estructura dinámica del modelo conduciría a estimaciones no eficientes de la relación a largo plazo y posiblemente a invalidar el análisis. La razón de utilizar un retardo de cuatro tiempos en el regresor desfasado se debe a las características de los datos utilizados, ya que se utilizan datos trimestrales, y no anuales como los análisis empíricos previos; por lo tanto, el retardo cuatro supone el mismo efecto trimestral que el retardo uno en datos anuales.

La variable Inflación aparece en numerosos modelos, como los de Deaton (1978), Davidson (1978) y Fuster (1993). La interpretación que dan a la 
introducción de esta variable es que puede recoger posibles efectos de ilusión monetaria. Es decir, la incertidumbre que provoca la tasa de inflación puede tener efectos positivos en el ahorro y negativos en el consumo. Por lo tanto su exclusión pudiera sesgar el análisis.

\section{Modelos de Contraste Particulares: de Equivalencia Ricardiana y Keynesiano}

Como se menciona en el apartado anterior, la ventaja de la función estructural de consumo utilizada es que recoge tanto los modelos de Equivalencia Ricardiana como el Keynesiano. Entonces, dichas concepciones terminan siendo casos particulares de la ecuación (1), lo que permite contrastar en el caso especifico de México, cual tiene mayor grado de veracidad.

Para el caso particular de la concepción de la HER, y de acuerdo al modelo general (1), la restricción de Equivalencia Ricardiana débil supone que el efecto sobre el consumo que ejerce el déficit público es el mismo que el de los ingresos públicos. Por lo tanto, los coeficientes de las variables ingresos públicos y déficit, deben ser iguales en valor absoluto y significativamente distintos de cero. De modo que la restricción seria ${ }_{3}={ }_{4}=-{ }_{2}$, y en consecuencia el modelo de Equivalencia Ricardiana débil queda:

$$
\Delta C P_{t}=\beta_{0}+\beta_{i} C P_{t-4}+\beta_{2} Y_{t}+\beta_{3} I P_{t}+\beta_{4} D P_{t}+\beta_{5} \pi_{t}+U_{t}
$$

Donde $\mathrm{G}$ es el gasto público (G=IP+DP). Para cumplir este modelo particular, su coeficiente ${ }_{3}$ debe ser negativo y significativo.

$\mathrm{El}$ modelo de equivalencia fuerte seria aquel que satisface la restricción anterior, y adicionalmente también $\alpha_{4}+\alpha_{3}=-\alpha_{2}$. Es decir, la combinación lineal de los coeficientes de ingresos públicos y del déficit deben ser igual al coeficiente del ingreso de las economías domesticas pero de signo inverso. En otras palabras, para que el modelo fuerte de Equivalencia Ricardiana se cumpla el efecto del ingreso privado y del gasto público deben ser de igual magnitud (pero de signo contrario). Por lo tanto, el modelo de fuerte queda:

$$
\Delta C P_{t}=Y_{0}+Y_{i} C P_{t-4}+Y_{2}\left(Y_{t}-G_{t}\right)+Y_{3} \pi_{t}+U_{t}
$$

Para caso particular keynesiano, el modelo de consumo se obtendría al imponer dos restricciones al modelo general: la primera, que el coeficiente del 
ingreso privado, sea igual, en valor absoluto, al de los ingresos públicos. Y la segunda, que el déficit no resulte significativo. Es decir, $\alpha_{4}=0$ y $\alpha_{2}=-\alpha_{3}$. Por lo tanto, el modelo de consumo keynesiano queda:

$$
\Delta C P_{t}=O_{0}+O_{\mathrm{i}} C P_{t-4}+O_{2}\left(Y_{t}-I P_{t}\right)+O_{3} \pi_{t}+U_{t}
$$

Una vez especificado el modelo general de partida y los modelos particulares que provienen del general, tanto ricardiano como keynesiano, solo resta probar los modelos y obtener los resultados de acuerdo al contraste de ambas concepciones teóricas.

Resultados obtenidos de la estimación

Para llevar a cabo la estimación, se evalúa la especificación (1) desde el punto de vista de la teoría de la cointegración de Granger (1987). Por lo tanto se utiliza el procedimiento bietápico de Engle-Granger(1987), el cual consiste en estimar primero la relación de cointegración por Mínimos Cuadrados Ordinarios (MCO) y, posteriormente, estimar un Mecanismo Corrector de Error (MCE) introduciendo los residuos de la relación de cointegración estimada, desfasados un periodo. Esta estimación bietápica se utiliza debido a que supone la convergencia entre las relaciones de largo plazo con la de corto plazo para determinar el contraste de la HER con la función de consumo estructural propuesta.

Los datos utilizados abarcan del periodo de 1993 hasta el 2007 trimestrales; la razón de utilizar solo hasta el 2007 es debido al corto periodo que el Instituto Nacional de Estadística Geografía e Informática (INEGI) utiliza para contabilizar el consumo privado con la nueva metodología. Las variables Ingreso, consumo y las cuentas fiscales han sido deflactadas a precios de 1993 y, como ya se comento con anterioridad, en el caso de la inflación se tomo la anualizada para tener mayor eficiencia en la estimación.

Siguiendo el método de estimación propuesto, y utilizando el modelo general (1) para contrastar las concepciones tanto keynesiana como ricardiana, al testear el orden de integración de las variables estudiadas desde el enfoque de Dickey Fuller se observan los siguientes resultados: 
Cuadro 1

Prueba de Estacionariedad Dicky Fuller*

\begin{tabular}{|c|c|c|c|c|c|}
\hline Variable & $\mathrm{CP}$ & $\mathrm{Y}$ & $\mathrm{IP}$ & $\mathrm{DP}$ & $\boldsymbol{\pi}$ \\
\hline $\begin{array}{c}\text { Estadístico } \\
\mathrm{t} * *\end{array}$ & -13.96535 & -5.037229 & -9.610814 & -11.9719 & -4.030802 \\
& $(0.0000)$ & $(0.0001)$ & $(0.0000)$ & $(0.0000)$ & $(0.0002)$ \\
\hline Orden de & $\mathrm{I}(1)$ & $\mathrm{I}(1)$ & $\mathrm{I}(1)$ & $\mathrm{I}(1)$ & $\mathrm{I}(1)$ \\
Integración & & & & & \\
\hline
\end{tabular}

*Series en primeras diferencias

**Los valores en paréntesis representan los valores $\mathrm{P}$.

Al probar que las variables no tienen raíz unitaria, y se vuelven estacionarias al diferenciarlas una vez (ósea son de orden uno), se prueba que las variables cointegran. Por lo tanto, la combinación lineal entre ellas necesariamente será de orden I(0) y siguiendo la metodología Engle-Granger, se procede a estimar el modelo de manera estática por MCO para encontrar la relación de largo plazo, obteniendo los siguientes parámetros estimados:

Cuadro 2

Estimación de la Función de Consumo de largo plazo*

\begin{tabular}{|c|c|c|c|c|}
\hline CP & Y & IP & DP & $\boldsymbol{\pi}$ \\
\hline & 0.374288 & 0.650153 & 0.072782 & -1577.513 \\
& $(0.0000)$ & $(0.2271)$ & $(0.7879)$ & $(0.0002)$ \\
\hline
\end{tabular}

*Los valores en paréntesis representan los valores $\mathrm{P}$.

Estos resultados muestran que el ingreso y la inflación son significativos, lo que nos indica teóricamente que el consumo depende positivamente del ingreso y negativamente de la inflación. Se observa que las variables fiscales no son significativas, lo que puede advertir el no cumplimiento de la HER en el largo plazo. Pero para conocer el contraste de los modelos particulares se utiliza el estadístico F de Wald, para comprobar si las múltiples restricciones que se desprenden del modelo general se aceptan o se rechazan. Los resultados de la prueba $\mathrm{F}$ de Wald se describen en la tabla siguiente: 


\section{Cuadro 3}

Prueba F de Wald para las restricciones de los modelos particulares (largo plazo)

\begin{tabular}{|c|c|c|c|}
\hline Modelo Particular & $\begin{array}{c}\text { Equivalencia Ricar- } \\
\text { diana Débil }\end{array}$ & $\begin{array}{c}\text { Equivalencia Ricar- } \\
\text { diana Fuerte }\end{array}$ & Keynesiano \\
\hline $\begin{array}{c}\text { Estadístico F de } \\
\text { Wald* }\end{array}$ & 3.827892 & 22.20952 & 2.145369 \\
$(0.0284)$ & $(0.0000)$ & $(0.1277)$ \\
\hline
\end{tabular}

*Los valores en paréntesis representan los valores $\mathrm{P}$.

Como se puede observar, las hipótesis planteadas al modelo general (1) se aceptan solo en el caso keynesiano para el largo plazo. Al mostrar un estadístico significativo mayor al 95\% de confianza, se concluye que la concepción teórica keynesiana en el caso del largo plazo es aceptada. En lo que respecta a la concepción de Equivalencia Ricardiana fuerte se observa que esta no es significativa en el lago plazo, y para la concepción débil de la misma tampoco se puede afirmar que esta sea significativa. Por lo tanto, se concluye que los consumidores mexicanos, en el largo plazo, no toman en cuenta el financiamiento gubernamental a la hora de consumir.

Para analizar lo que respecta al corto plazo, y siguiendo el criterio de Engle-Granger (1987), se realiza un Modelo Corrector de Errores (MCE), en el cual las estimaciones resultantes fueron las siguientes:

\section{Cuadro 4}

Estimación de la Función de Consumo de Corto Plazo*

\begin{tabular}{|c|c|c|c|c|c|}
\hline$\Delta \mathrm{CP}$ & $\Delta \mathrm{Y}$ & $\Delta \mathrm{IP}$ & $\Delta \mathrm{DP}$ & $\Delta \varpi$ & $\mathrm{MCE}(-1)$ \\
\hline & 0.183516 & 0.392815 & -0.334374 & -4024.531 & -0.745056 \\
& $(0.0060)$ & $(0.3706)$ & $(0.1225)$ & $(0.0000)$ & $(0.0000)$ \\
\hline
\end{tabular}

*Los valores en paréntesis representan los valores $\mathrm{P}$.

Los coeficientes estimados con las series diferenciadas presentan ciertas singularidades: en primer lugar, se obtienen parámetros significativos para el ingreso y para la inflación, tal como sucede en el largo plazo. En segundo lugar, se puede observar que aunque los coeficientes de los ingresos públicos y del déficit no son significativos, son relativamente iguales en valor 
absoluto, tal como argumenta las hipótesis planteadas en el modelo general, esto puede evidenciar la aceptación de la concepción débil de la HER. Con esto, además, los resultados pudieran converger con la concepción fuerte ricardiana. Para contrastar lo anterior se realiza la misma prueba F de Wald para comprobar la contundencia de las restricciones impuestas al modelo en el corto plazo:

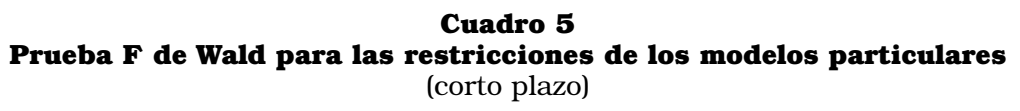

\begin{tabular}{|c|c|c|c|}
\hline Modelo & Equivalencia & Equivalencia & Keynesiano \\
Particular & Ricardiana Débil & Ricardiana Fuerte & \\
\hline Estadístico F & 1.222216 & 7.60587 & 2.56436 \\
de Wald* & $(0.3036)$ & $(0.0003)$ & $(0.0875)$ \\
\hline
\end{tabular}

*Los valores en paréntesis representan los valores $\mathrm{P}$.

Al observar los estadísticos de prueba para las restricciones impuestas al modelo se puede observar que la concepción keynesiana se cumple, pero también la ricardiana aunque solo en su versión débil, por lo tanto no se puede afirmar claramente cual concepción teórica es la dominante en el corto plazo, pero el resultado en el caso de la Equivalencia Ricardiana puede ser ensombrecido por dos motivos: el primero es el hecho de que su concepción fuerte no es significativa; el segundo es el hecho de que los coeficientes no son significativos en el modelo general de corto plazo, aunque estos si presenten características singulares que hacen que la restricción débil pueda cumplirse. Por lo tanto, si bien no se puede rechazar la concepción de la HER en su versión débil en el corto plazo, no tiene la contundencia necesaria que tienen los coeficientes en el caso de la concepción keynesiana.

Como conclusión se establece que la HER en el caso México no se cumple en el largo plazo, y en el corto plazo no se cuenta con una línea específica para afirmar o desechar esta concepción en su versión débil. Para el caso del contraste keynesiano se observa que se cumple en ambas especificaciones temporales, pero tiene el mismo problema en el corto plazo: converge con la concepción ricardiana débil, lo que genera un resultado ambiguo. 


\section{Conclusiones}

Después de un análisis desde las perspectivas teórica y empírica se concluye que la HER tiene ciertas particularidades que pueden impedir su efectivo cumplimiento en alguna economía. Autores han mencionado diversos motivos por los que la HER no se puede cumplir y han hecho investigación empírica para sustentar sus argumentos.

De acuerdo al análisis empírico hecho para México se concluye que los consumidores no tienden a preocuparse por el financiamiento gubernamental para consumir, al menos en el largo plazo. El consumidor mexicano tiende a creer que un aumento del déficit representa un efecto riqueza, ya que aumentan las transferencias y por lo tanto aumenta el consumo, tal como lo menciona la teoría keynesiana.

En el análisis de corto plazo el resultado es ambiguo, no existen argumentos necesarios para desestimar la concepción de la HER versión débil o de la teoría keynesiana, aunque hay ligera tendencia en aceptar con mayor énfasis la concepción keynesiana que la ricardiana debido a que su versión fuerte no fue significativa, y además los parámetros fiscales no fueron significativos en el modelo general de corto plazo.

Estos resultados son importantes ya que representan un estudio formal sobre la función de un déficit publico en relación al consumo en México, por lo tanto, el planeador social puede flexibilizar el uso del déficit esperando que, al ser mayores las transferencias, aumente el ingreso de las economías domesticas, lo que aumentará el consumo, lo que aumentará la demanda agregada, la producción y el crecimiento económico.

\section{Bibliografía}

Barro Robert J., Are Government Bonds Net Wealth?, Journal of Political Economy 81, 1974, pp. 1095-1117.

Barro, Robert, J., Macroeconomía: teoría y politica, Madrid, Mc Graw Hill. 1997.

Barro, Robert J., Reply to Feldstein and Buchanan, Journal of Political Economy 84, (1976), pp 343- 350.

Bernheim, B. Douglas, Ricardian Equivalence: An Evaluation of Theory and Evidence, Massachusetts, Macroeconomics Annual, 1987, pp. 263-304.

Feldstein, M. S., Perceived Wealth in Bonds and Social Security: A Comment, Journal of Political Economy 84, 1976, pp. 331-360. 
Feldstein, M. S. y Elmendorf, D. W., Taxes, budget deficit and consumer spending: some new evidence, Massachusetts, National Bureau of Economic Research, Working Paper, núm. 2355, 1987.

Fuster, M., La Hipótesis de Equivalencia Ricardiana: Un Análisis Empírico en los Países de la Comunidad Europea, Barcelona, Investigaciones Económicas, vol. XVII, 1993, pp. 495-506.

Kochin, L., Are Future Taxes Anticipated by Consumers?: Comment, vol. 6, núm. 3, 1974. pp. 385-394.

Kormendi, Roger C., Government Debt, Government Spending, and Private Sector Behavior, The American Economic Review, vol. 73, núm. 5, 1983. pp. 994-1010.

Mankiw, N. Gregory, Macroeconomics, New York, Worth, 2009.

Marchante, A. Consumo Privado y Gasto Público: Evidencia para la Economía Española. Universidad de Málaga, Revista de Economía Aplicada, Numero 1(vol. 1), 1993. pp.125-149.

Romer, David H., Macroeconomía avanzada, Madrid; McGraw-Hill Interamericana, 2006.

Tello, C., Sobre la Reforma Tributaria en México, EconomíaUNAM, núm. 7, 2010. pp. 39-56.

Tobin, J. y Buiter, W., Fiscal and monetary policies, capital formation, and economic activity, en: The government and capital formation, cap. 3. Cambridge, Ballinger Press, 1980.

Yawitz, J. B. y Meyer, L. H., An empirical investigation of the extent of tax discounting, Journal of Money, Credit and Banking, 8, 1976. pp. 247254. 